\title{
Early diagnosis of venous thromboembolism as a clinical primary symptom of occult cancer: Core proteins of a venous thrombus (Review)
}

\author{
SIWAN WEN ${ }^{1}$, QIANGLIN DUAN ${ }^{1}$, FAN YANG $^{2}$, GUIYUAN LI $^{3}$ and LEMIN WANG $^{1}$ \\ Departments of ${ }^{1}$ Cardiology, ${ }^{2}$ Clinical Laboratory and ${ }^{3}$ Oncology, \\ Tongji University Affiliated to Tongji Hospital, Shanghai 200065, P.R. China
}

Received July 18, 2015; Accepted March 3, 2017

DOI: $10.3892 / \mathrm{ol} .2017 .6175$

\begin{abstract}
Malignancy is one of the risk factors of venous thromboembolism (VTE). As a common accompanying factor of malignant tumors, almost $20 \%$ of idiopathic VTEs are identified in patients with occult types of cancer as the primary symptom. The type of internal association that exists between malignant tumors and VTE has not yet been determined. The present review discusses the following: i) Reversible combinations between core proteins of venous thrombi and their ligand proteins. With the condition of immune cell balancing function collapse, which is characterized as dysfunction immune cells and impaired immune functions, the human body loses the function of eliminating infectious/malignant cells quickly and effectively. Thus, integrins $\beta 2$ and $\beta 3$ on the membrane of platelets and white blood cells are activated to combine with fibrinogen ligands to form an intravenous mesh-like structure, which acts as an intravenous biological filter that prevents infectious/malignant cells from flowing back into the circulatory system. During the defense process, blood cells (mainly red blood cells) stagnate and fill the filter, which results in venous thrombotic diseases. ii) Tumor cells, which cannot be eliminated quickly, proliferate and invade; or ischemic necrosis destroys peripheral tissues and vessels (veins and arteries), resulting in the formation of a biological filter in injured veins. The filter is filled with stranded tumor cells, which prevents the hemorrhagic metastasis of malignant cells. The formation of an intravenous biological filter results from the transition of the body's own defense capabilities, which is also a physical/histopathological phenomenon. iii) An increase in the number of core proteins in a venous thrombus is a basic molecular step in the formation of intravenous biological
\end{abstract}

Correspondence to: Professor Lemin Wang, Department of Cardiology, Tongji University Affiliated to Tongji Hospital, 389 Xincun Road, Shanghai 200065, P.R. China

E-mail: wanglemin@tongji.edu.cn

Key words: venous thromboembolism, occult cancer, core protein, integrin, intravenous biological filter, specific diagnosis filters, which is also defined as a marker of the newly initiated defensive barrier. Increased levels of integrins $\beta 1, \beta 2$ and $\beta 3$ are useful in not only the specific diagnosis of VTE, but also in the early recognition of occult malignant tumors in idiopathic VTE patients.

\section{Contents}

1. Introduction

2. Pathology of acute venous thrombosis

3. Major proteins and core proteins of an acute venous thrombus

4. Localization of core proteins in an acute venous thrombus at a cellular level

5. Establishing biological filters during acute venous thrombosis

6. Inevitability of activation of an intravenous physical defense line

7. Increased integrin $\beta 1, \beta 2$ and $\beta 3$ levels in patients with malignant tumors

8. Conclusion

\section{Introduction}

Venous thromboembolism (VTE) includes pulmonary thromboembolism (PE) and deep venous thrombosis. VTE that can be diagnosed in clinics is known as dominant VTE. The clinical spectrum of VTE is relatively wide, since VTE can occur in different organs and tissues. However, dormant VTE, which is hard to diagnose in clinics, is often revealed upon autopsy. PE has become a global medical care problem due to its high morbidity, misdiagnosis and mortality rates $(1,2)$. VTE can be divided into two categories: Genetic VTE and acquired VTE. According to the results of epidemiological investigations, the incidence of genetic VTE is relatively low, with the majority of the VTEs being acquired VTEs (3). The two types can be termed as symptomatic VTE when it is hard to distinguish between them (3).

The American College of Chest Physicians published 9 editions of guidelines for VTE diagnosis, treatment and 
prevention (4) between 1995 and 2012. Proposed risk factors include advanced age, infection, malignancy, autoimmune disease, surgery, trauma, pregnancy, long-trip syndrome and family history.

Malignancy is one of the risk factors of VTE. The prevalence of VTE in patients with malignancy is 4-7 times higher than that of patients without malignancy $(5,6)$. In addition, the survival rate in patients with malignancies and thrombi is 2-3 times lower than that of cancer patients without any thrombus (7). Furthermore, $10-25 \%$ of patients with idiopathic VTE as the first symptom are subsequently diagnosed with cancer within 2 years, with the majority of the diagnoses formed within 6 months, and the incidence of thrombosis within 6 months of diagnosis being 4 times higher than that of other periods (8). VTE is not only a common complication of malignant tumors, but also the second leading cause of mortality in cancer patients (9).

Certain issues with regard to VTE require addressing, such as why patients with malignant tumors are often also affected by VTE, why idiopathic VTE is always an early symptom of occult cancer and the association between them.

Wang et al (10) reported that VTE is an inevitable product in the proliferation phase of cancer cells, and acts as a physical barrier by preventing cancer cells from hemorrhagic metastasis. Therefore, there is necessarily a connection between malignant tumors and the occurrence of VTE. To highlight the association between malignant tumors and VTE, a novel mechanism of VTE must be discussed.

\section{Pathology of acute venous thrombosis}

It has previously been reported (11) that the acute venous thrombus taken from a pulmonary artery with a catheter presents as a red thrombus to the naked eye, which is characterized as easily degraded. The red thrombus is comprised of red blood cells, platelets, white blood cells and plasma proteins, as shown by microscopy (Fig. 1).

\section{Major proteins and core proteins of an acute venous thrombus}

Plasma proteins are important components that combine with blood cells to form the material basis of thrombi. In a previous study, mass spectrographic analyses demonstrated that a large majority of the proteins identified were fibrinogen; the remaining proteins included fibrin and cytoskeletal proteins (12). Thrombi containing fibrinogen degrade easily due to reversible combinations between fibrinogen and their ligand proteins, which theoretically explains why an acute venous thrombus is easy to autolyze, why delayed thrombolysis is effective and why it is easy to lyse the thrombus through interventional fragmentation (12).

An acute venous thrombus, also known as a red thrombus, is composed of red blood cells, platelets, white blood cells and fibrinogen. The manner in which fibrinogen binds to blood cells during the formation of a venous thrombus is representative of the mechanism of acute venous thrombosis. The use of tandem mass spectrometry and the bioinformatic analysis of emboli in patients with acute PE has revealed that integrin subunits $\beta 1$, $\beta 2$ and $\beta 3$ are the core proteins of the emboli (Fig. 2).

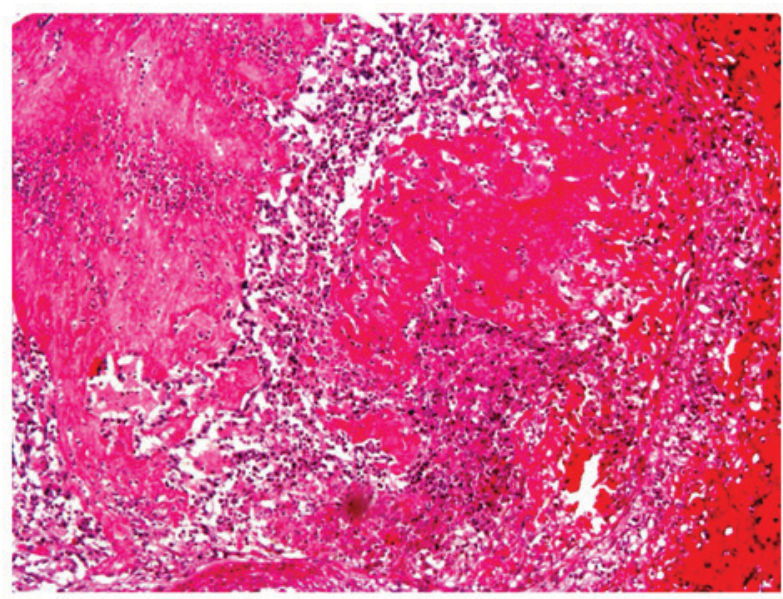

Figure 1. Hematoxylin and eosin staining of thrombus demonstrating that a venous thrombus is a red thrombus in which massive red blood cells and white blood cells with dark-brown nuclei aggregate (magnification, x200).

Integrins are important members of the cell adhesion molecule family, mediating adhesion between cells, and between cells and the extracellular matrix (ECM), with involvement in bidirectional signaling transduction between cells and the ECM. Integrins can combine to their different ligands in various cellular processes, namely the physical or pathological processes of angiogenesis, invasion, metastasis, inflammation, wound healing and coagulation (13).

Integrin is a transmembrane heterodimer composed of subunits $\alpha$ and $\beta$ at a ratio of 1:1. To date (Fig. 3A), a total of $18 \alpha$ subunits and $8 \beta$ subunits have been identified, and these can form 24 functional heterodimers, which may be classified into 8 groups $(\beta 1-\beta 8)$ based on the $\beta$ subunit. In the same group, the $\beta$ subunit is identical, but the $\alpha$ subunit is distinct. At rest, the $\alpha$ subunit is covered by the $\beta$ subunit and thus the integrin is unable to bind to ligands. Following activation, the extension of the $\beta$ subunit exposes the $\alpha$ subunit. The $\alpha$ subunit mainly mediates the specific and reversible binding between integrins and their ligands (Fig. 3B), and the $\beta$ subunit dominates the signal transduction and regulation of the affinity of integrins (14-16).

\section{Localization of core proteins in an acute venous throm- bus at a cellular level}

The $\beta 1$ subunit exists mainly on lymphocytes and platelets, and its ligands include laminin, collagen, thrombospondin, fibronetin and vascular cell adhesion molecule-1. The $\beta 2$ subunit is mainly distributed on neutrophils and monocytes, and its ligands include fibrinogen, intracellular adhesion molecule (ICAM), factorX and ic $3 b$. The $\beta 3$ subunit is mainly observed on platelets, and its ligands include fibrinogen, fibronetin, vitronectin, von Willebrand factor (vWF) and thrombospondin (17-19).

Wang et al (11) collected thrombi through a catheter attached to the pulmonary artery of patients with acute PE. Immunohistochemistry revealed that dark-brown integrin $\beta 1$ was expressed on the lymphocytes, but that no expression of laminin, fibronectin, collagen-I or collagen-II was observed on the lymphocytes. Dark-brown integrin $\beta 2$ was expressed on 


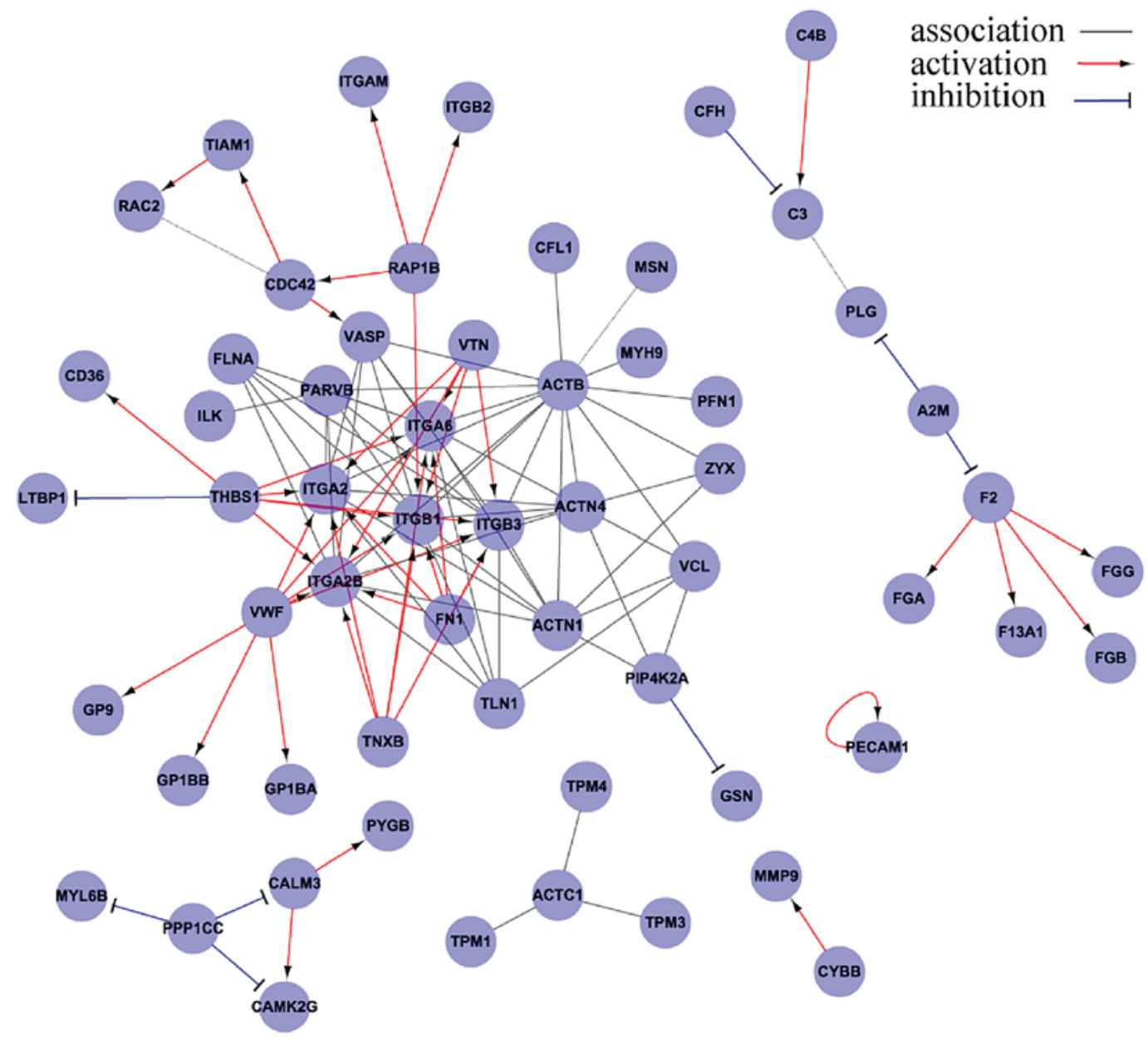

Figure 2. Tandem mass spectrometry and bioinformatic analysis of emboli in patients with acute pulmonary embolism. Integrin subunits $\beta 1, \beta 2$ and $\beta 3$ are core proteins of the emboli.

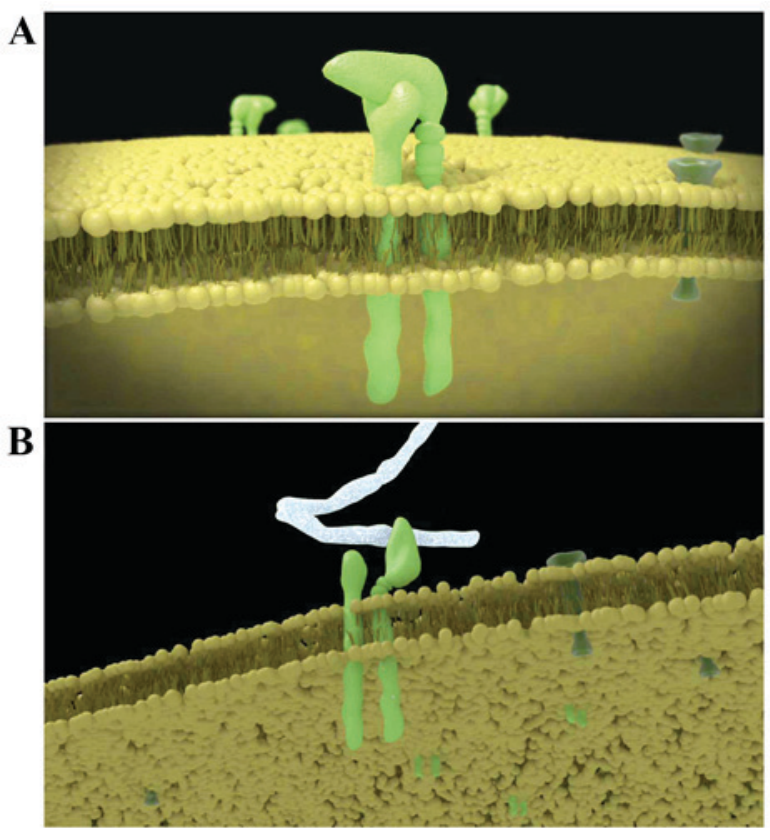

Figure 3. (A) Mode pattern of the integrin on the cell membrane. Integrin is a transmembrane heterodimer composed of subunits $\alpha$ and $\beta$ at a ratio of 1:1. At rest, the $\alpha$ subunit is covered by the $\beta$ subunit and thus the integrin is unable to bind to ligands. (B) Following activation, the extension of the $\beta$ subunit exposes the $\alpha$ subunit. The $\alpha$ subunit mainly mediates the specific and reversible binding between integrins and their ligands. the neutrophils, which bound to fibrinogen. ICAM, factor X and $\mathrm{iC} 3 \mathrm{~b}$ were expressed on the neutrophils, and dark-brown integrin $\beta 3$ was expressed on the platelets, which aggregated to become a thrombotic skeleton with a coral-like structure; these platelets bound fibrinogen to create a mesh-like structure (Fig. 4A), which is similar to artificial vena cava filters and was termed an intravenous biological filter in the study. No expression of fibronectin, vitronectin or vWF was observed on the platelets, and dark-brown factor Xa was distributed on the mesh-like structure, which was composed of fibrin/fibrinogen.

Another study reported that the expression of subunits $\beta 1$, $\beta 2$ and $\beta 3$ increased significantly in patients with VTE (20). ROC curve analysis was used to assess the diagnostic performance of these proteins in 120 VTE patients. The area under the curve (AUC) of integrins $\beta 1, \beta 2$ and $\beta 3$ in the VTE patients was $0.870,0.821$ and 0.731 , respectively. Optimum cutoffs of integrins $\beta 1, \beta 2$ and $\beta 3$ calculated according to Youden's index were $10.29,91.10$ and $10.35 \mathrm{pg} / \mathrm{ml}$, respectively. With these optimum cutoffs, the sensitivity, specificity, and positive and negative predictive values were as follows: For integrin $\beta 1$, $80.3,83.7,71.1$ and $89.3 \%$, respectively; for integrin $\beta 2,78.6$, 73.7, 59.4 and $87.6 \%$, respectively; and for integrin $\beta 3,68.4$, $71.2,54.3$ and $81.8 \%$ respectively. The combined AUC of the three integrins was 0.916 , and the sensitivity, specificity, and positive and negative predictive values were $84.6,90.8,81.7$ 

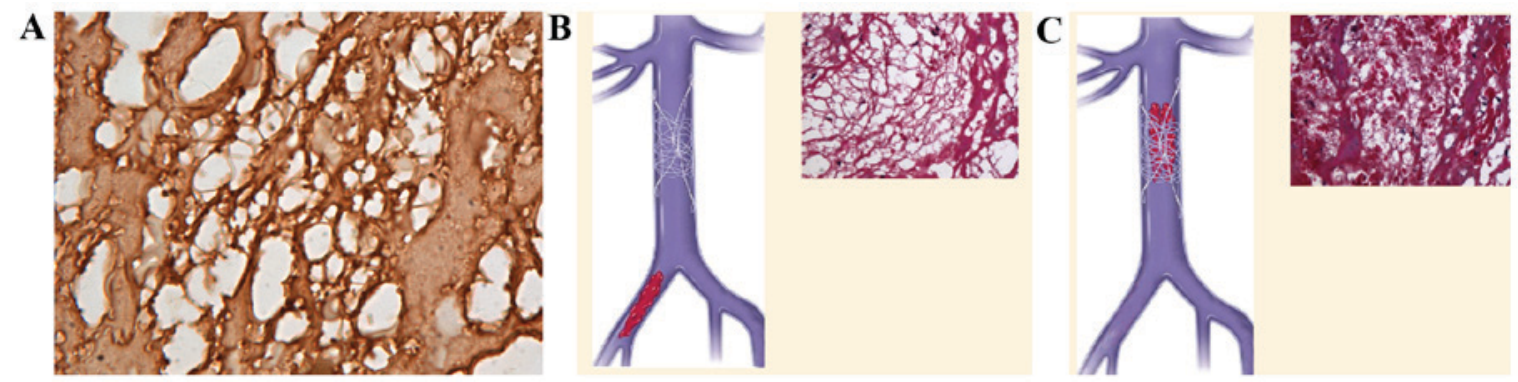

Figure 4. (A) Immunohistochemistry demonstrating that platelets, which aggregate to become a thrombotic skeleton, bind fibrinogen to construct a mesh-like structure (anti-fibrinogen antibody; 1:100 dilution; magnification, $\mathrm{x} 1,000$ ), which is similar to artificial vena cava filters and known as an intravenous biological filter. (B) (Left) Mode pattern of artificial nest-like inferior vena cava filter. (Inset) Mesh-like structure is a nest-like biological filter in veins under the microscope (magnification, x400, Masson staining). (C) (Left) Mode pattern of artificial nest-like inferior vena cava filter filled with thrombi. (Inset) Nest-like biological filters formed inside a venous thrombus under the microscope, in which red blood cells are the dominant blood cells found (magnification, $\mathrm{x} 400$, Masson staining). Inset images reproduced with permission (11).
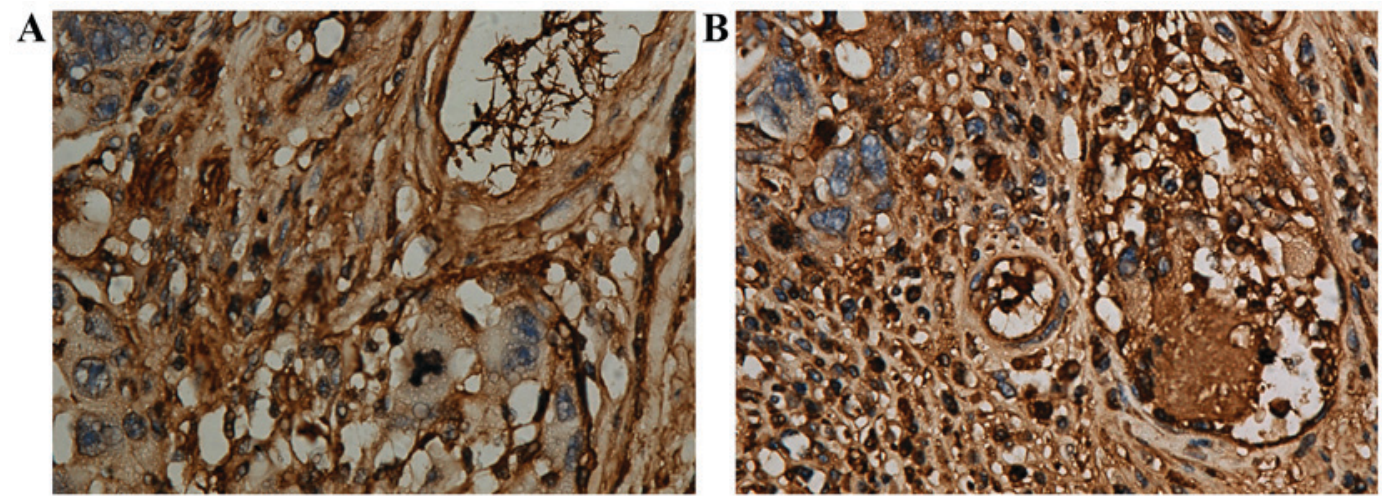

Figure 5. (A) In colon cancer, a mesh-like structure (anti-fibrinogen antibody; 1:100 dilution; magnification, $x 400$ ) was observed in venules (right upper area of this image), and cancer cells were observed in this mesh-like structure (anti-fibrinogen antibody; 1:100 dilution; magnification, x400). The mesh-like structure was a nest-like biological filter. (B) Pathological findings of cancer tissues revealing cancer cells. An intravenous biological filter (right area of the image) was filled with cancer cells (anti-fibrinogen antibody; 1:100 dilution; magnification, x400). Inset images reproduced with permission (11).

and $92.0 \%$, respectively. A clinical study confirmed that the VTE patients with significantly increased expression of integrins $\beta 1, \beta 2$ and $\beta 3$ also exhibited relatively high specificity and sensitivity (20).

\section{Establishing biological filters during acute venous thrombosis}

Different types of artificial nest-like inferior vena cava filters have been used in clinics for 30 years, the mechanism of which prevents the genesis of PE by blocking the deep venous thrombi flowing back to the pulmonary arteries through the filter (Fig. 4A). Two core proteins of thrombi, integrins $\beta 2$ and $\beta 3$, bind their ligand fibrinogen to construct a mesh-like structure, which becomes a nest-like biological filter in the veins (Fig. 4B and C) (11).

As a precise and perfect life entity, the human body is constantly regulating itself towards balance and stability. The production of intravenous biological filters is a result of the self-regulation of the human body.

Xiong et al (16) identified biological filters in the veins of resected sigmoid colon adenocarcinoma tissues (Fig. 5A). Malignant cancer cells were identified in biological filters, which impeded the hematogenous metastasis of the cancer cells (Fig. 5B).

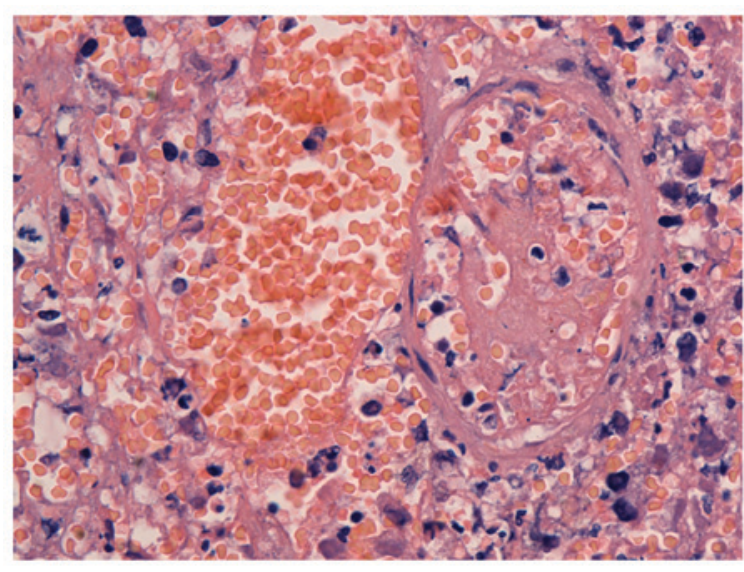

Figure 6. Necrotic region in poorly-differentiated gastric carcinoma presenting with exudation of a large number of red blood cells.

\section{Inevitability of activation of an intravenous physical de- fense line}

The defense system of the human body is the immune system. Simply speaking, it is the function of the immune system to remove all foreign agents inside the human body, including external pathogenic microorganisms, implants, invasive foreign 
bodies and toxins from wounds, and internally generated senile and malignant cells (10).

The genesis of malignancy indicates the loss of innate and adaptive immune cell balancing functions. In other words, the occurrence of cancer means a collapse of the immune cell balancing function, which means a loss of the function of immune clearance of malignant cells. When the immune cell balancing function collapses, it is a basic principle to start using reserved immune functions. Thus, an alternative defensive barrier is activated (10).

The proliferation speed of cancer cells usually exceeds that of small vessels (veins and arteries), which could easily cause ischemic necrosis, and thus increased permeability of vessels and the destruction of small vessels. In addition, the proliferation of malignant tumor cells and invasion of surrounding small vessels can also cause the destruction of small vessels (10). Wang et al (10) identified a biological filter in the veins of resected sigmoid colon adenocarcinoma tissue (Fig. 5A). Malignant cancer cells were revealed in the biological filter, which impeded the hematogenous metastasis of the cancer cells (Fig. 5B). It was found that (10) fibrinogen formed a mesh-like structure in the peripheral small veins of a resected sigmoid colon adenocarcinoma, which was filled with cancer cells, thus preventing their translocation. When cells escaped from the mesh-like structure, this presented as metastasis of the tumor cells. The formation of a biological filter in the surrounding veins of malignant tumor tissue is a replacement or supplement to the loss of immune function to prevent hematogenous metastasis. The occurrence of VTE in patients with malignancy is the first stage of physical immune defense. Patients with malignant tumors bleed easily. Wang et al (10) also identified exudation of a large number of red blood cells out of vessels (Fig. 6) and the accumulation of large amounts of fibrinogen in necrotic regions of poorly-differentiated gastric carcinoma, indicating destruction of small vessels and/or the increased permeability of vessels.

According to a study on the biopsies of malignant tumor patients, $50 \%$ of such individuals were affected by VTE prior to mortality (21). From the genesis and development of malignant tumor cells, and the morphological characteristics of the proliferation stage, the present study speculated that almost every patient with a malignant tumor has the possibility of having VTE, and that almost every patient with a malignant tumor has the possibility of having bleeding, due to the processes of tumor cell proliferation, the destruction of peripheral tissues and vessels, and the establishment of a defensive barrier, which are physical/pathological phenomena of the human body. However, VTE and bleeding of the peripheral tissues around cancer cells are hard to recognize at an early stage.

\section{Increased integrin $\beta 1, \beta 2$ and $\beta 3$ levels in patients with malignant tumors}

Song et al (22) reported that the levels of the integrin $\beta 1$, $\beta 2$ and $\beta 3$ core proteins of thrombi increased in patients with malignant tumor cells, among which, integrins $\beta 1$ and $\beta 3$ increased significantly. The relative risks of integrins $\beta 1, \beta 2$ and $\beta 3$ in malignant tumor patients were 1.655, 1.314 and 1.852 times that of a control group, respectively, while the combined risk of increased integrins $\beta 1, \beta 2$ and $\beta 3$ in the malignant tumor group was 4.895 times that of a control group, indicating that integrins $\beta 1, \beta 2$ and $\beta 3$ are the molecular basis of the increased risk of VTE in patients with cancer.

There are significant differences in the therapy, risk of reoccurrence and survival period between cancerous and non-cancerous VTEs. If patients with malignant tumors are able to receive an early diagnosis due to early warning signals from VTE occurrence, precious time may be saved for early treatment, which could have a significant impact on the VTE and tumors. Based on the aforementioned data, CG144 guidelines published by the National Institute for Health and Clinical Excellence (NICE) in 2012 (https://www.nice.org. uk/guidance/cg144) have begun to recommend that idiopathic VTE patients $>40$ years old should be screened for tumors in order to exclude occult tumors (23); this has been declared to be a milestone in the treatment and prevention of VTE by Shaboodien et al (21).

\section{Conclusion}

Clinical tests to detect core proteins of venous thrombi, presenting as increased levels of integrins $\beta 1, \beta 2$ and $\beta 3$, are useful not only in the diagnosis of VTE, but also in the early recognition of occult malignant tumors in idiopathic VTE patients.

\section{References}

1. Piazza G and Goldhaber SZ: Physician alerts to prevent venous thromboembolism. J Thromb Thrombolysis 30: 1-6, 2010.

2. Cardiovascular Disease Educational and Research Trust; Cyprus Cardiovascular Disease Educational and Research Trust; European Venous Forum; International Surgical Thrombosis Forum; International Union of Angiology; Union Internationale de Phlébologie: Prevention and treatment of venous thromboembolism. International Consensus Statement (guidelines according to scientific evidence). Int Angiol 25: 101-161, 2006.

3. Heit JA: The epidemiology of venous thromboembolism in the community. Arterioscler Thromb Vasc Biol 28: 370-372, 2008.

4. Guyatt GH, Akl EA, Crowther M, Gutterman DD and Schuunemann HJ; American College of Chest Physicians Antithrombotic Therapy and Prevention of Thrombosis Panel: Executive summary: Antithrombotic Therapy and Prevention of Thrombosis, 9th ed: American college of chest physicians evidence-based clinical practice guidelines. Chest 141 (2 Suppl): 7S-47S, 2012.

5. Falanga A and Russo L: Epidemiology, risk and outcomes of venous thromboembolism in cancer. Hamostaseologie 32: 115-125, 2012.

6. Seddighzadeh A, Shetty R and Goldhaber SZ: Venous thromboembolism in patients with active cancer. Thromb Haemost 98: 656-661, 2007.

7. Khorana AA, Ahrendt SA, Ryan CK, Francis CW, Hruban RH, $\mathrm{Hu}$ YC, Hostetter G, Harvey J and Taubman MB: Tissue factor expression, angiogenesis, and thrombosis in pancreatic cancer. Clin Cancer Res 13: 2870-2875, 2007.

8. Kakkar AK: Cancer-associated thrombosis. Br J Cancer 102 (Suppl 1): S1, 2010.

9. Goldhaber SZ and Bounameaux H: Pulmonary embolism and deep vein thrombosis. Lancet 379: 1835-1846, 2012.

10. Wang LM, Duan QL, Yi XH, Zeng Y, Gong Z and Yang F: Venous thromboembolism is a product in proliferation of cancer cells. Int J Clin Exp Med 7: 1319-1323, 2014.

11. Wang LM, Duan QL, Yang F, Yi XH, Zeng Y, Tian HY, Lv W and Jin Y: Activation of circulated immune cells and inflammatory immune adherence are involved in the whole process of acute venous thrombosis. Int J Clin Exp Med 7: 566-572, 2014. 
12. Wang L, Gong Z, Jiang J, Xu W, Duan Q, Liu J and Qin C: Confusion of wide thrombolytic time window for acute pulmonary embolism: Mass spectrographic analysis for thrombus proteins. Am J Respir Crit Care Med 184: 145-146, 2011.

13. Giancotti FG and Ruoslahti E: Integrin signaling. Science 285 1028-1032, 1999.

14. Humphries MJ: Integrin structure. Biochem Soc Trans 28: 311-339, 2000

15. Takada Y, Ye X and Simon S: The integrins. Genome Biol 8: 215, 2007.

16. Xiong JP, Stehle T, Diefenbach B, Zhang R, Dunker R, Scott DL, Joachimiak A, Goodman SL and Arnaout MA: Crystal structure of the extracellular segment of integrin alpha Vbeta3. Science 294: 339-345, 2001.

17. Gerber DJ, Pereira P, Huang SY, Pelletier C and Tonegawa S: Expression of alpha $\mathrm{v}$ and beta 3 integrin chains on murine lymphocytes. Proc Natl Acad Sci USA 93: 14698-14703, 1996.

18. Lityńska A, Przybyło M, Ksiazek D and Laidler P: Differences of alpha3beta1 integrin glycans from different human bladder cell lines. Acta Biochim Pol 47: 427-434, 2000.
19. Solovjov DA, Pluskota E and Plow EF: Distinct roles for the alpha and beta subunits in the functions of integrin alphaMbeta2. J Biol Chem 280: 1336-1345, 2005.

20. Song Y, Yang F, Wang L, Duan Q, Jin Y and Gong Z: Increased expressions of integrin subunit $\beta 1, \beta 2$ and $\beta 3$ in patients with venous thromboembolism: New markers for venous thromboembolism. Int J Clin Exp Med 7: 2578-2584, 2014.

21. Shaboodien R, Stansby G, Hunt BJ and Agarwal R: Unprovoked venous thromboembolism: Assess for cancer. Lancet Oncol 13: 973-974, 2012.

22. Song Y, Wang L, Yang F, Li G, Duan Q and Gong Z: Increased expressions of integrin subunit $\beta 1, \beta 2$ and $\beta 3$ in patients with cancer-correlation analysis between risk factors of VTE and expression of core proteins. Int J Clin Exp Med 8: 2772-2777, 2015

23. Chong LY, Fenu E, Stansby G and Hodgkinson S; Guideline Development Group: Management of venous thromboembolic diseases and the role of thrombophilia testing: Summary of NICE guidance. BMJ 344: e3979, 2012. 